\title{
Prevalencia de fasciolosis en bovinos de Costa Rica (2014). Comparación de cuatro técnicas diagnósticas
}

\author{
Rojas-Araya, D. ${ }^{1}$; Montero, A. ${ }^{2}$; León, D. ${ }^{3}$; Romero, J. ${ }^{4}$ \\ ${ }^{1,2.3}$ Centro de Investigación en Enfermedades Tropicales, Facultad de Microbiología, Universidad de Costa Rica, \\ Sede Rodrigo Facio, Apartado Postal: 11501-2060 San José, Costa Rica. ${ }^{1,4}$ Maestría en Epidemiología, Posgrado \\ Regional en Ciencias Veterinarias Tropicales, Escuela de Medicina Veterinaria, Universidad Nacional, Campus \\ Benjamín Núñez, Apartado Postal: 86-3000 Heredia, Costa Rica. \\ E-mail: diana.rojas_a@ucr.ac.cr
}

\begin{abstract}
Resumen
Rojas-Araya, D.; Montero, A.; León, D.; Romero, J.: Prevalencia de fasciolosis en bovinos de Costa Rica (2014). Comparación de cuatro técnicas diagnósticas. Rev. vet. 27: 2, 80-85, 2016. Se realizó un estudio transversal con el objetivo de determinar la prevalencia de fasciolosis en bovinos sacrificados en mataderos de Costa Rica y comparar cuatro técnicas diagnósticas disponibles (inspección macroscópica de hígados, sedimentación estándar de heces, detección de coproantígeno en heces y sedimentación estándar del líquido de la vesícula biliar). Se trabajó con dos estratos de probabilidad de infección (alto y bajo). Tomando en cuenta todas las técnicas diagnósticas, se encontraron 11 muestras positivas del estrato de alta probabilidad [prevalencia 4,3\% (intervalo de confianza IC del 95\%:1,8-6,7)] y 1 del estrato de baja probabilidad [prevalencia $0,5 \%$ (IC 95\%: 0,0-1,4)]. Al comparar los métodos diagnósticos, tomando el decomiso de hígados como método de referencia, se obtuvieron valores de sensibilidad y especificidad de 40 y $100 \%$ para la sedimentación estándar de heces, 50 y $100 \%$ para la detección de coproantígeno en heces (ELISA), y 70 y 99,8\% para la sedimentación estándar del líquido de la vesícula biliar, respectivamente. Se concluye que la inspección macroscópica de hígados en los mataderos es una buena herramienta de vigilancia de la enfermedad y que esta parasitosis se distribuye de manera heterogénea en el país.
\end{abstract}

Palabras clave: bovino, Fasciola hepatica, prevalencia, diagnóstico.

\begin{abstract}
Rojas-Araya, D.; Montero, A.; León, D.; Romero, J.: Prevalence of fasciolosis in cattle of Costa Rica (2014). Comparison of four techniques for its diagnosis. Rev. vet. 27: 2, 8085 , 2016. A cross-sectional study was done to determine the prevalence of fasciolosis in cattle slaughtered in abattoirs of Costa Rica and to compare 4 different diagnostic techniques (macroscopic examination of the livers, standard sedimentation of stool, standard sedimentation of the fluid of the gallbladder, and detection of copro-antigen in fecal samples. The sample was divided into two strata of probability of infection (high and low). Considering all the techniques, 11 positive samples from the stratum of high probability were found [prevalence $4.3 \%$ (confidence interval CI 95\%: 1.8-6.7)] and 1 of the stratum of low probability [prevalence 0.5\% (CI 95\%: 0.0-1.4)]. When comparing all diagnostic methods, considering the liver condemnation as the reference method, values of sensitivity and specificity respectively of 40 and $100 \%$ for the standard sedimentation of stool, 70 and $99.8 \%$ for the standard sedimentation of the fluid of the gallbladder and 50 and $100 \%$ for the ELISA that detects copro-antigen in feces were obtained. It is concluded that the macroscopic examination of livers in slaughterhouses is a good tool for the monitoring of the disease and that this parasite is distributed heterogeneously in the country.
\end{abstract}

Key words: cattle, Fasciola hepatica, prevalence, diagnostic efficiency.

\section{INTRODUCCIÓN}

Fasciola hepatica es un parásito natural de los rumiantes y el agente causal de la fasciolosis. Esta enfer-

Recibido: 7 marzo 2016 / Aceptado: 4 julio 2016 medad tiene una distribución cosmopolita y es responsable de provocar cuantiosas pérdidas económicas ${ }^{24}$. El diagnóstico tradicional de esta parasitosis se basa en la observación de huevecillos del parásito en las heces del animal infectado. Sin embargo, muchos países efectúan el registro de la infección en sus bovinos a tra- 
vés de la inspección post-mortem de las vísceras en los mataderos y del decomiso de hígados infectados con ejemplares adultos del parásito ${ }^{27}$.

La inspección post-mortem del líquido de la vesícula biliar en búsqueda de huevecillos, también ha sido empleada como método diagnóstico, mediante técnicas de sedimentación ${ }^{24}$. Alternativamente, pueden utilizarse pruebas inmunológicas, ya sea para la detección de productos metabólicos del trematodo localizado en los ductos biliares, los cuales son liberados y expulsados en las heces, o bien para la búsqueda de anticuerpos contra el parásito en sangre o leche ${ }^{20}$.

Los datos sobre prevalencia de fasciolosis en América Latina varían en función del método diagnóstico utilizado, época del año y localidad evaluada. En dicha zona, la distribución del parásito es amplia, habiendo sido reportado en México, Costa Rica, Colombia, Venezuela, Brasil, Perú, Bolivia, Argentina, Chile, Ecuador, Uruguay y Paraguay; así también en algunas islas caribeñas como Cuba, Puerto Rico, República Dominicana, Santa Lucía, Jamaica, Guadalupe y Martinica ${ }^{22}$.

En Costa Rica se reconoce la presencia de este parásito en las provincias de Cartago y Limón 2, 4,7, 10, 15,21 . Estimaciones realizadas en fincas han señalado tasas de infección del $17 \%{ }^{15}, 42 \%{ }^{10}$ y $11 \%{ }^{3}$ en ganado bovino. En tres mataderos del área metropolitana de Costa Rica, la prevalencia estimada a partir de registros de decomiso de hígados infectados con el parásito (20002006) fue de un $0,8 \%{ }^{2}$.

Debido a la escasez de datos acerca de la prevalencia de este parásito en diferentes zonas de Costa Rica, el objetivo de la investigación fue determinar tal prevalencia en reses provenientes de diversas regiones, así como contrastar los resultados de diferentes técnicas diagnósticas en reses infectadas naturalmente, y compararlas con la herramienta de vigilancia disponible en Costa Rica que es el registro oficial de decomisos de hígados infectados en mataderos.

\section{MATERIAL Y MÉTODOS}

Se realizó un estudio observacional transversal, para determinar la prevalencia de Fasciola hepatica en el hato bovino nacional sacrificado en tres de los cuatro mataderos de clase A de Costa Rica. Se utilizó un muestreo estratificado debido a que los estudios llevados a cabo en el país y la experiencia obtenida por los veterinarios regentes de los mataderos, señalan que la infección por $F$. hepatica no es uniforme en el país.

Los mataderos de clase A se utilizaron como sitios de colecta de las muestras debido a que del total de reses sacrificadas en el país, un $82 \%$ de las mismas se procesan en estos tres mataderos de clase $\mathrm{A}^{13}$, los cuales cumplen con las regulaciones nacionales y sacrifican hembras y machos provenientes de todo el país.

Se trabajó con la hipótesis de que el ganado bovino que proviene de las regiones Huetar Atlántica (HA) y Central Metropolitana (CM) es el más expuesto al parásito (estrato de alta probabilidad EAP), y que el resto de las regiones son de baja probabilidad para adquirir la infección (estrato de baja probabilidad EBP): reses provenientes de las regiones Central Occidente (CO), Central Sur (CS), Chorotega (CH), Pacífico Central (PC), Brunca (B) y Huetar Norte (HN).

El tamaño de la muestra por estrato se calculó con el programa WinEpiscope, sobre una población de 1.972.882 cabezas de ganado, que constituyen aproximadamente el hato nacional. Para poder estimar una prevalencia de hasta $25 \%$ en el EAP, con un nivel de confianza del $95 \%$ y un error aceptado entre $5-6 \%$ se tomó una muestra de 260 individuos. En el EBP, para poder calcular una prevalencia no mayor al $5 \%$, con un nivel de confianza del $95 \%$ y un margen de error del $3 \%$, se tomó una muestra de 205 individuos. Ambos estratos sumaron una muestra total de 465 reses.

El número total de reses a evaluar por estrato se distribuyó en proporción al número de cabezas de ganado por región que constituya ese estrato ${ }^{18}$. Específicamente, del estrato de alta probabilidad se tomaron 185 de la región HA y 75 de CM. Del estrato de baja probabilidad 12 de la región $\mathrm{CO}, 9$ de $\mathrm{CS}, 55$ de $\mathrm{CH}, 18$ de PC, 33 de B y 78 de HN.

Los mataderos fueron visitados durante los meses de setiembre y octubre del 2014. Las reses fueron escogidas al azar, según su zona de procedencia. Las guías de movilización y la lista de inspección ante mortem de bovinos (documentos oficiales del Servicio Nacional de Salud Animal, SENASA) se utilizaron para obtener la información sobre el sexo y la región de procedencia de cada animal. Se excluyeron del estudio reses menores a 6 meses o que provinieran de otros países.

A cada res escogida se le inspeccionó el hígado, se colectó líquido de la vesícula biliar y se tomó una muestra de heces. Cada una de las tres muestras se transportó a $4^{\circ} \mathrm{C}$ y se procesó en el Laboratorio de Helmintología Médica de la Universidad de Costa Rica. Tales muestras fueron colectadas post-mortem, para no interferir con el funcionamiento de la planta de sacrificio ni infringir lesiones al animal.

La inspección macroscópica inicial de los hígados se hizo directamente en la planta de sacrificio, y consistió en la revisión visual y mecánica de los conductos hepáticos en búsqueda de las calcificaciones típicas asociadas con la enfermedad, así como la presencia del parásito. Aquellos hígados que presentaron parásitos fueron transportados al laboratorio, donde los conductos hepáticos fueron escindidos para recuperar más parásitos adultos. Para el análisis de las vesículas biliares, se tomó una muestra de $40 \mathrm{ml}$ de bilis previamente agitada y se utilizó un método de sedimentación estándar ${ }^{24}$.

En el caso de las muestras de heces, se recolectaron $50 \mathrm{~g}$ de cada res. A partir de la misma se efectuó la detección del coproantígeno de $F$. hepatica por medio de un ELISA y se realizó una técnica de sedimentación estándar utilizando $4 \mathrm{~g}$ de heces ${ }^{12}$. Para la detección de coproantígeno de $F$. hepatica en heces, las muestras fueron preservadas a temperatura de $-20^{\circ} \mathrm{C}$ hasta su 
análisis ${ }^{8}$ y luego procesadas según las especificaciones del fabricante, por medio de un ELISA indirecto tipo sándwich que detecta coproantígenos de $F$. hepatica (Bio-X Bovine Fasciola Hepatica Antigen Elisa Kit ${ }^{\circledR}$, Bio-X Diagnostics, Bélgica) ${ }^{6}$.

Los datos y características demográficas fueron tabulados en una hoja de Microsoft Excel e importados al programa estadístico IBM SPSS Statistics 22 para su análisis. Se calculó la prevalencia estimada de la enfermedad, con sus intervalos de confianza al $95 \%$ (IC $95 \%$ ), tanto a nivel global como por estrato, y para cada tipo de método diagnóstico. La prueba de Chi-cuadrado $\left(\chi^{2}\right)$ se realizó para determinar la asociación entre la infección y las variables sexo y región de procedencia del animal (asociación estadísticamente significativa si $\mathrm{p}<0,05$ ).

Para cada técnica diagnóstica se calculó su sensibilidad, especificidad y valor predictivo positivo y negativo, utilizando la inspección macroscópica de los hígados como método de referencia. Adicionalmente, para evaluar la concordancia entre las pruebas, se calculó el índice de kappa. Este índice fue catalogado de acuerdo a la valoración propuesta por Landis y Koch ${ }^{17}$ : si el valor es menor a 0,00 el grado de acuerdo es "sin acuerdo", entre 0,00-0,20 es "insignificante", entre 0,21-0,40 es "mediano", entre 0,41-0,60 es "moderado", entre $0,61-0,80$ es "sustancial" y entre $0,81-1,00$ es "casi perfecto". Todos los estimadores se calcularon al 95\% de confianza, utilizando el programa Win Episcope.

\section{RESULTADOS Y DISCUSIÓN}

Se muestrearon 73 reses de las 75 propuestas para la región $\mathrm{CM}$ del estrato de alta probabilidad, por lo cual en definitiva se trabajó con un total de 258 reses para el EAP y un total de 463 muestras para ambos estratos. Del total de reses muestreadas, 294 (63\%) fueron hembras; aspecto que concuerda parcialmente con el hecho de que en el inventario total del ganado en el país (2012), se reportó un $71,5 \%$ de hembras ${ }^{18}$. Debido a ello es dable esperar que al final de la cadena productiva también se registre mayor cantidad de hembras en los establecimientos de sacrificio.

La prevalencia del parásito varió de acuerdo a la zona de probabilidad de la que provenía la res, al sexo del animal y al método empleado para el diagnóstico. Tomando en cuenta todas las técnicas diagnósticas, se encontraron 12 muestras positivas, 11 de reses provenientes del estrato de alta probabilidad [prevalencia de 4,3\% (IC 95\%: 1,8- 6,7)] y 1 en el estrato de baja probabilidad [prevalencia de 0,5\% (IC 95\%: 0,0-1,4)]. Esto reveló una diferencia estadísticamente significativa ( $\mathrm{p}$ : $0,004)$ por estrato de procedencia de las reses.

La distribución por regiones dentro de los estratos fue de 6 reses positivas dentro de la región CM (5 en HA y 1 en B). Este hallazgo coincide con lo encontrado en otras investigaciones realizadas en el país, donde se reportó la presencia del parásito en la zona de Cartago y Limón, provincias que constituyen casi la totalidad de las regiones $\mathrm{CM}$ y HA, respectivamente. En un estudio llevado a cabo en bovinos durante 1976 y 1977 en Turrialba, se reportó una frecuencia del parásito del $3,4 \%{ }^{1}$, valor cercano al encontrado en este estudio.

Frecuencias más altas se hallaron a nivel nacional al evaluar fincas específicas con presencia del parásito, como un caso en el cual se reportó $17 \%$ de infección por $F$. hepatica en bovinos de carne de una finca en Agua Caliente de Cartago ${ }^{15} ; \mathrm{u}$ otro donde se encontró un $42 \%$ de infección en bovinos de leche en fincas de Santa Cruz, cantón de Turrialba ${ }^{10}$. De igual forma, en una finca en Siquirres de Limón, otros investigadores encontraron que durante agosto de 2005 a agosto de 2006, la prevalencia de fasciolosis determinada por la presencia de huevecillos en heces, fue del $11,3 \%{ }^{3}$.

Es importante considerar que en estos parásitos de ciclo complejo, las condiciones climáticas y la época del año en que se realice el muestreo pueden incidir en los resultados obtenidos. El cúmulo de datos sobre $F$. hepatica en el país señala que es posible que haya regiones donde probablemente su presencia se beneficie por condiciones ambientales favorables al desarrolle del ciclo, tales como precipitaciones pluviales, características del suelo, luz y temperatura, que permitan la sobrevivencia y tasa reproductiva del hospedero intermediario y de las formas evolutivas de vida libre del parásito.

En Costa Rica, tanto la Provincia de Limón (región HA), como la parte oriental de la Provincia de Cartago (de Turrialba hacia el Este y parte de la región CM), forman parte de la región climatológica conocida como "Región Caribe". En esta zona de clima tropical húmedo ${ }^{25}$, la precipitación promedio es cercana a los 3300 $\mathrm{mm}$ anuales y se reconoce como una región sin período seco definido, cuya temperatura promedio es alrededor de $24-26^{\circ} \mathrm{C}^{16}$. Es posible que estas condiciones ambientales favorezcan a los hospederos intermediarios y promuevan el desarrollo del ciclo del parásito, sin embargo, hace falta investigar más sobre las plantas y moluscos hospederos involucrados en estos sitios y los factores ecológicos asociados a los mismos.

Un posible sesgo en este estudio fue que de la región central metropolitana se muestrearon al azar solamente 3 reses provenientes del cantón de Turrialba. Esta condición puede haber respondido a la presencia de un matadero que haya captado las reses de la zona y que, además, ésta es un área dedicada principalmente a la producción de ganado lechero, por lo que la regularidad con la que sacrifican reses es menor en comparación a otras zonas ${ }^{18}$. Se resalta esta particularidad debido a que, anteriormente, muchos de los estudios efectuados en el país sobre este parásito se llevaron a cabo en Turrialba, y es una zona que se ha reportado como endémica para la enfermedad. Este matadero no se contempló dentro de los muestreos debido a que no es de clase A y por legislación nacional, no cuenta con un veterinario regente ni inspectores de SENASA.

Por otra parte, el hallazgo de una res con $F$. hepatica proveniente de la región Brunca podría ser reflejo de 
la movilización interna de ganado bovino que se da dentro del país ${ }^{13}$, ya que según información emanada de entidades oficiales, la mayor importación de reses a nivel nacional a la región Brunca proviene de las regiones $\mathrm{PC}$ y $\mathrm{CM}$, siendo esta última uno de los sitios con presencia evidenciada del parásito.

Desde el punto de vista de la salud pública, los casos de fasciolosis en seres humanos se relacionan con las zonas donde la enfermedad en bovinos es común. En este estudio, el hallazgo del parásito en reses de determinadas zonas del país, concuerda con los pocos casos publicados en Costa Rica de la enfermedad en personas, los cuales han sido San Ramón de Tres Ríos, Santa Teresa de Capellades de Cartago y Turrialba ${ }^{4,9,15}$. Sin embargo, es importante mencionar que el sistema nacional de salud carece, en general, de métodos diagnósticos específicos $\mathrm{y}$ de rutina para esta parasitosis y su hallazgo en la mayoría de los casos es casual, principalmente durante intervenciones quirúrgicas de vías biliares.

Con respecto al sexo de los animales, de las 12 muestras positivas por al menos alguna de las técnicas diagnósticas, 11 reses fueron hembras y solo en un macho se reportó la infección (p: 0,015), aspecto que coincide con lo reportado en otras investigaciones realizadas en diferentes países ${ }^{14,23,26}$ y en donde se asocia con los sistemas de producción, debido a un mayor tiempo de permanencia de las hembras en pastos, por las prácticas asociadas a la cría de bovinos y producción lechera y por ende más probabilidad de exponerse a plantas con la metacercaria ${ }^{5}$.

En cuanto a la comparación de los métodos diagnósticos empleados y la prevalencia encontrada con cada uno de ellos, de las muestras positivas provenientes del EAP (IC 95\%) surge que la mayoría de hallazgos positivos ocurrieron al utilizar la inspección macroscópica del hígado y la menor cantidad de muestras positivas se encontraron al analizar las muestras de heces por sedimentación estándar (Tabla 1).

La matriz de concordancia entre los diferentes métodos diagnósticos empleados, que evidencia el coeficiente kappa, reveló que todas las pruebas tuvieron un valor de coeficiente kappa mayor a 0 , es decir que la concordancia observada no es la que se espera por causa exclusiva del azar ${ }^{17}$. El mayor valor de coeficiente kappa se obtuvo al comparar la inspección macroscópica de hígados y el análisis de la vesícula biliar, grado de acuerdo entre pruebas catalogado "acuerdo sustancial" ${ }^{17}$ (Tabla 2).
La comparación entre pruebas, utilizando el decomiso de hígados en los establecimientos como método de referencia, permitió comparar la herramienta diagnóstica utilizada actualmente en el país con otras usualmente empleadas en otros contextos (Tabla 3).

Esta comparación es importante porque en Costa Rica, al igual que en muchos países, se lleva el registro de la infección de $F$. hepatica en sus bovinos a través de la inspección post-mortem de las vísceras en los mataderos y el decomiso de hígados infectados con el parásito. En este estudio, a diferencia de otras investigaciones $^{24,27}$, la inspección del hígado resultó ser sensible y concordante con lo encontrado al usar los otros métodos.

La inspección post-mortem del líquido de la vesícula biliar fue la técnica que presentó la mayor concordancia con el decomiso de hígados. Aunque la sensibilidad obtenida en este caso fue menor a la reportada por otros investigadores ${ }^{24}$, se destaca su alta especificidad y practicidad en comparación con la búsqueda de los huevecillos en las heces, ya que su colecta y procesamiento es más sencillo.

La observación de huevecillos del parásito en las heces del animal infectado reportó valores bajos de sensibilidad que podrían aumentarse analizando mayor cantidad de heces y efectuando análisis por triplicado ${ }^{24}$; sin embargo, aún solamente procesando $4 \mathrm{~g}$ de una muestra, es altamente laboriosa en su montaje y análisis ${ }^{12}$. Con respecto a la prueba inmunológica que 
Tabla 3. Comparación de métodos diagnósticos de $F$. hepatica y valores de sensibilidad, especificidad y predictibilidad con respecto al método de referencia (decomiso de hígado).

\begin{tabular}{|c|c|c|c|}
\hline \multirow{2}{*}{ método a comparar } & \multirow{2}{*}{ decomisos } & \multicolumn{2}{|c|}{ total } \\
\hline & & decomisado & no decom. \\
\hline \multicolumn{4}{|l|}{ SE líquido VB } \\
\hline presencia huevecillos & 7 & 1 & 8 \\
\hline ausencia huevecillos & 3 & 452 & 455 \\
\hline total & 10 & 453 & 463 \\
\hline sensibilidad (\%) & 70,0 & & \\
\hline especificidad (\%) & 99,8 & & \\
\hline valor predic. $+(\%)$ & 87,5 & & \\
\hline valor predic. neg. (\%) & 99,3 & & \\
\hline \multicolumn{4}{|l|}{ Coproantígeno ELISA } \\
\hline muestras heces posit. & 5 & 0 & 5 \\
\hline muestras heces negat. & 5 & 453 & 458 \\
\hline total & 10 & 453 & 463 \\
\hline sensibilidad (\%) & 50,0 & & \\
\hline especificidad (\%) & 100,0 & & \\
\hline valor predic. $+(\%)$ & 100,0 & & \\
\hline valor predic. - (\%) & 98,9 & & \\
\hline \multicolumn{4}{|l|}{ SE de heces } \\
\hline presencia huevecillos & 4 & 0 & 4 \\
\hline ausencia huevecillos & 6 & 453 & 459 \\
\hline total & 10 & 453 & 463 \\
\hline sensibilidad (\%) & 40,0 & & \\
\hline especificidad (\%) & 100,0 & & \\
\hline valor predic. $+(\%)$ & 100,0 & & \\
\hline valor predic. - (\%) & 98,7 & & \\
\hline
\end{tabular}

SE: sedimentación estándar. VB: vesícula biliar. Valor predictivo: confianza del 95\%.

detecta productos metabólicos liberados por el trematodo y expulsados en las heces ${ }^{20}$, tiene como ventaja ser rápida, fácil y capaz de detectar antígenos en heces antes del período prepatente ${ }^{19}$; sin embargo, en este estudio, la sensibilidad comparada con la inspección macroscópica del hígado fue de un 50\%.

Otros estudios bajo condiciones experimentales de infección controlada han reportado valores de sensibilidad y especificidad de $94 \%$ y $100 \%{ }^{20}$, y de $94 \%$ y 93\% ${ }^{11}$ al utilizarse en bovinos con infección natural. Una particularidad aquí observada fue que de las 12 muestras positivas por al menos uno de los métodos, 6 muestras resultaron positivas por este método según el punto de corte estipulado por la casa comercial; sin embargo, 3 muestras más presentaron valores de absorbancia mayores al resto de las muestras negativas pero ligeramente por debajo del punto de corte, y por ende se reportaron como negativas. Estas 3 muestras fueron positivas también por al menos otros dos métodos más, razón por la que no se sospecha de su positividad y se cree que es posible que revalorando el punto de corte de la prueba éstas hubiesen dado positivas. Resultados similares fueron obtenidos en otras investigaciones ${ }^{8}$.

Se concluye que la metodología utilizada en los mataderos clase A de Costa Rica para registrar la presen- cia de $F$. hepatica es válida, y que esto puede responder a la adecuada capacitación técnica de los veterinarios e inspectores intervinientes. Por otra parte, surge que la fasciolosis debe considerarse como enfermedad prioritaria en algunas regiones del país y encararse el rastreo de fincas problema para controlarla, distribuyendo adecuadamente los recursos y acciones para contener y eliminar los riesgos potenciales a la salud humana y animal, así como reducir las pérdidas económicas asociadas con esta parasitosis.

\section{REFERENCIAS}

1. Alice E. 1978. Fasciolosis humana y bovina. Datos relativos a fasciolosis humana y bovina en Costa Rica. Rev Méd Costa Rica 45: 117-120.

2. Alpízar C. 2008. Infección por Fasciola hepatica en bovinos de carne de una finca en Siquirres e impacto económico del decomiso de hígados en tres mataderos de Costa Rica. Trabajo final de graduación, Univ. Nac. Costa Rica, $40 \mathrm{p}$.

3. Alpízar C, Bianque J, Jiménez A, Hernández J, Berrocal A, Romero J. 2013. Fasciola hepatica en ganado bovino de carne en Siquirres y lesiones anatomo-histopatológicas de hígados bovinos decomisados en mataderos de Costa Rica. Agron Costarricense 37: 7-16.

4. Arroyo R, Mora J, Molina S, Troper L, Amador A. 1981. Fascioliasis hepática humana en Costa Rica. Rev Costarricense Cienc Med 2: 35-57.

5. Avcioglu H, Guven E, Balkaya I, Kaynar O, Hayirli A. 2014. Evaluation of coprological and serological techniques for diagnosis of bovine fasciolosis. Isr J Vet Med 69: 203-210.

6. Bio-X Diagnostic. Bio-X bovine Fasciola hepatica antigen ELISA kit (sandwich indirect assay for faeces): kit for antigenic diagnosis. http://www.biox.com

7. Brenes R, Arroyo G, Muñoz G, Delgado E. 1968. Estudio preliminar sobre Fasciola hepatica en Costa Rica. Rev Biol Trop 15: 137-142.

8. Brockwell Y, Spithill T, Anderson G, Grillo V, Sangster N. 2013. Comparative kinetics of serological and coproantigen ELISA and faecal egg count in cattle experimentally infected with Fasciola hepatica and following treatment with triclabendazole. Vet Parasitol 196: 417-426.

9. Castro J. 1948. Fascioliasis hepática humana en Costa Rica. Presentación de un caso. Rev Cub Med Trop 4: 160161.

10. Chang E, Cartín M. 1983. Diagnóstico y control de la fasciolosis bovina en el distrito de Santa Cruz de Turrialba, provincia de Cartago. Trabajo final de graduación, Escuela Med. Vet., Univ. Nac. Costa Rica, 49 p.

11. Charlier J, De Meulemeester L, Claerebout E, Williams D, Vercruysse J. 2008. Qualitative and quantitative evaluation of coprological and serological techniques for the diagnosis of fasciolosis in cattle. Vet Parasitol 153: 44-51.

12. Conceiçao M, Durao M, Costa I, Correia J. 2002. Evaluation of a simple sedimentation method (modified McMaster) for diagnosis of bovine fascioliasis. Vet Parasitol 105: 337-343. 
13. Díaz A, Calvo B, Trelles S. 2013. La rastreabilidad del ganado bovino: experiencia de Costa Rica. En: Desarrollo e implementación de un sistema de control de movimiento de ganado bovino en Costa Rica, publ. del Instituto Interamericano de Cooperación para la Agricultura IICA, San José, Costa Rica, p. 61.

14. Góngora R. 2006. Prevalencia de Fasciola hepatica en bovinos faenados en el matadero municipal de La Paz. Tesis de Grado Med. Vet., Univ. Auton. G.R.Moreno (UAGRM), Santa Cruz de la Sierra, Bolivia, 46 p.

15. Herrera R. 1979. Diagnóstico de fasciolosis: estudio comparativo entre la intradermoreacción, exámen coproparasitológico y contrainmunoelectroforesis. Trabajo final de graduación Med. Vet., Universidad Nacional Costa Rica, $19 \mathrm{p}$.

16. Instituto Meteorológico Nacional (IMN). 2008. El clima, su variabilidad y cambio climático en Costa Rica. En: Proyecto Segunda Comunicación Nacional sobre Cambio Climático en Costa Rica. San José, Costa Rica, p. 75.

17. Landis J, Koch G. 1977. The measurement of observer agreement for categorical data. Biometrics 33: 159-174.

18. Madrigal J, Fallas M. 2013. Informe encuesta ganadera 2012. Publ. Corporación Ganadera (CORFOGA), San José, Costa Rica, 72 p.

19. Martínez J, Robles D, Rojo F, Martínez M. 2012. Comparison of three different techniques to diagnose Fasciola hepatica infection in experimentally and naturally infected sheep. Vet Parasitol 190: 80-86.

20. Mezo M, González M, Carro C, Ubeira F. 2004. An ultrasensitive capture ELISA for detection of Fasciola hepatica coproantigens in sheep and cattle using a new monoclonal antibody (MM3). J Parasitol 90: 845-852.
21. Mora J, Arroyo R, Molina S, Troper L, Irías E. 1980. Nuevos aportes sobre el valor de la fasciolina. Estudio en un área endémica de Costa Rica. Bol Ofic Sanit Panam 89: 409-414.

22. Morales G, Pino L. 2004. Fasciola hepatica y distomatosis hepática bovina en Venezuela. Publ. FAO (Red de helmintología para América Latina y el Caribe: contribución a la conferencia electrónica), $19 \mathrm{p}$.

23. Phiri A, Phiri K, Sikasunge C, Monrad J. 2005. Prevalence of fasciolosis in zambian cattle observed at selected abattoirs with emphasis on age, sex and origin. $J$ Vet Med B 52: 414-416.

24. Rapsch C, Schweizer G, Grimm F, Kohler L, Bauer C, Deplazes P, Braun U, Torgerson P. 2006. Estimating the true prevalence of Fasciola hepatica in cattle slaughtered in Switzerland in the absence of an absolute diagnostic test. Int J Parasitol 36: 1153-1158.

25. Solano J, Villalobos R. 2001. Aspectos fisiográficos aplicados a un bosquejo de regionalización geográfico climático de Costa Rica. Rev. Tópicos Meteorológicos y Oceanográficos 8: 26-39.

26. Soto J, Bohorquez N. 1984. Análisis epidemiológico de decomisos sanitarios en bovinos sacrificados en el estado Zulia. Rev Vet Venezolana 48: 12-29.

27. Yildirim A, Ica A, Duzlu O, Inci A. 2007. Prevalence and risk factors associated with Fasciola hepatica in cattle from Kayseri province, Turkey. Rev Med Vet Toulouse 158: 613-617. 